\title{
Acute Bacterial Meningitis in Qatar: A Hospital-Based Study from 2009 to 2013
}

\author{
Fahmi Yousef Khan, ${ }^{1}$ Mohammed Abu-Khattab, ${ }^{2}$ Eman Abdulrahman Almaslamani, ${ }^{3}$ \\ Abubaker Ahmed Hassan, ${ }^{2}$ Shehab Fareed Mohamed, ${ }^{4}$ Abdurrahman Ali Elbuzdi, ${ }^{5}$ \\ Nada Yagoub Elmaki, ${ }^{2}$ Deshmukh Anand, ${ }^{6}$ and Doiphode Sanjay ${ }^{6}$ \\ ${ }^{1}$ Department of Medicine, Hamad General Hospital, Doha, Qatar \\ ${ }^{2}$ Department of Medicine, Infectious Disease Division, Hamad General Hospital, Doha, Qatar \\ ${ }^{3}$ Department of Pediatrics, Infectious Disease Division, Hamad Medical Corporation, Sedra, Doha, Qatar \\ ${ }^{4}$ Hematology Division, Al Amal Hospital, Doha, Qatar \\ ${ }^{5}$ Medical Intensive Care Unit, Hamad General Hospital, Doha, Qatar \\ ${ }^{6}$ Department of Microbiology, Hamad General Hospital, Doha, Qatar
}

Correspondence should be addressed to Fahmi Yousef Khan; fakhanqal@gmail.com

Received 8 March 2017; Revised 10 May 2017; Accepted 16 May 2017; Published 13 July 2017

Academic Editor: Pere Domingo

Copyright (C) 2017 Fahmi Yousef Khan et al. This is an open access article distributed under the Creative Commons Attribution License, which permits unrestricted use, distribution, and reproduction in any medium, provided the original work is properly cited.

Background and Objectives. Bacterial meningitis is a common medical condition in Qatar. The aim of this study was to describe the clinical characteristics of bacterial meningitis, the frequency of each pathogen, and its sensitivity to antibiotics and risk factors for death. Patients and Methods. This retrospective study was conducted at Hamad General Hospital between January 1, 2009, and December 31, 2013. Results. We identified 117 episodes of acute bacterial meningitis in 110 patients. Their mean age was $26.4 \pm 22.3$ years (range: $2-74$ ) and $81(69.2 \%)$ of them were male patients. Fifty-nine episodes (50.4\%) were community-acquired infection and fever was the most frequent symptom (94\%), whereas neurosurgery is the most common underlying condition. Coagulase-negative staphylococci were the most common causative agent, of which $95 \%$ were oxacillin-resistant, while $63.3 \%$ of Acinetobacter spp. showed resistance to meropenem. The in-hospital mortality was 14 (12\%). Only the presence of underlying diseases, hypotension, and inappropriate treatment were found to be independent predictors of mortality. Conclusion. Acute bacterial meningitis predominantly affected adults and coagulase-negative staphylococci species were the common causative agent in Qatar with majority of infections occurring nosocomially. More than $90 \%$ of all implicated coagulase-negative staphylococci strains were oxacillin-resistant.

\section{Introduction}

Despite medical advances, acute bacterial meningitis (ABM) constitutes a global public health problem, especially in developing countries with poor health facilities due to high rates of malnutrition, poor living conditions, and lack of access to appropriate preventive and curative services that may predispose people to the disease and reduce their chances of receiving optimal treatment $[1,2]$. In developed countries, the burden of the disease has reduced and its epidemiology has changed as a result of the widespread use of vaccines against the most common meningeal pathogens [3].
Accurate information on important etiologic agents and populations at risk is needed to determine public health measures and ensure appropriate management of ABM [3]. In Qatar, although $\mathrm{ABM}$ is a common medical condition that physicians face, there are few reports describing this disease [4-6]. We conducted the present study, the purposes of which were to (1) describe the demographic and clinical characteristics of $\mathrm{ABM},(2)$ determine the relative frequency of each pathogen and its susceptibility to various antimicrobial agents, and (3) determine the outcome and the significant predictors of the outcome among patients with ABM in Qatar. 


\section{Materials and Methods}

2.1. Design and Setting. This retrospective descriptive study, which involved all in-patients with ABM, was conducted at Hamad General Hospital between January 1, 2009, and December 31, 2013. This hospital is a 603-bed tertiary care center that covers all specialties except for hematologyoncology, cardiology, and obstetrics and it has been Joint Commission International (JCI) accredited since 2006 and is the first hospital system in the region to achieve institutional accreditation from the Accreditation Council for Graduate Medical Education-International (ACGME-I). Currently, there are three adult ICUs in Hamad General Hospital, namely, Medical ICU (MICU) with 22 beds, Surgical ICU (SICU) with 12 beds, and Trauma ICU (TICU) with 15 beds.

2.2. Definitions. ABM was diagnosed on the basis of at least one of the following compatible clinical pictures with no other apparent cause: fever $\left(38^{\circ} \mathrm{C}\right)$, headache, meningeal signs, cranial nerve signs, and impaired mental status, plus one of the following $[7,8]$ :

(1) Positive cerebrospinal fluid (CSF) culture

(2) Positive CSF bacterial antigen test (with latex agglutination counterimmunoelectrophoresis) associated with pleocytosis mainly neutrophilic, defined as absolute $\mathrm{WBC} \geq 100$ cells $/ \mathrm{mm}^{3}$, with a decreased glucose level $\leq 40 \mathrm{mg} / \mathrm{dL}$ and an increased protein concentration $\geq 60 \mathrm{mg} / \mathrm{dL}$.

ABM was considered nosocomial if the diagnosis was made after more than 48 hours of hospitalization or within a short period of time (i.e., usually within one month after discharge from the hospital where the patient had received an invasive procedure, especially a neurosurgical procedure) [9]. On the other hand, ABM was considered as communityacquired if the diagnosis was made within the first 48 hours of hospitalization and the patient was not hospitalized in the preceding month [10]. Empirical antimicrobial therapy was deemed to be inappropriate if the antibiotics were administered more than 24 hours after CSF collection and/or when the dosage, route, and duration of treatment were not in accordance with hospital guidelines [11]. Hypotension was defined as blood pressure $<90 / 60 \mathrm{mmHg}$. Multidrugresistant organisms are defined as microorganisms, predominantly bacteria, that are resistant to one or more classes of antimicrobial agents [12].

Viral, fungal, mycobacterial, polymicrobial, and drug induced meningitis were excluded. ABM episodes with the same organism were included only once. Coagulase-negative staphylococci and viridans streptococci are considered as causative agents if CSF showed pleocytosis mainly neutrophilic, defined as absolute $\mathrm{WBC} \geq 100$ cells $/ \mathrm{mm}^{3}$, or a decreased glucose level $\leq 40 \mathrm{mg} / \mathrm{dL}$ or an increased protein concentration $\geq 60 \mathrm{mg} / \mathrm{dL}$. The primary outcome was inhospital mortality which included all causes of death during admission.
2.3. Isolation, Identification, and Antimicrobial Susceptibility Test of Microorganisms. Identification of isolates was based on colony morphology, Gram stain, oxidase, catalase, VITEK 2 Compact (bioMérieux, Durham, USA), and Phoenix (Becton Dickinson, NJ, USA). The antimicrobial minimal inhibitory concentrations (MICs) for the isolates were determined by using Phoenix (Becton Dickinson, NJ, USA) for GNB and staphylococci and enterococci (among Gram-positive cocci). For fastidious bacteria, susceptibility was determined with a gradient strip method (E-test strips, bioMérieux, Marcy-l'Étoile, France). The breakpoint interpretation was determined according to the recommendations of the Clinical Laboratory Standards Institute (CLSI) [13].

2.4. Source of Data and Data Collection. Cases were identified via hospital's discharge records, infection control records, and cerebrospinal fluid records maintained by the microbiology unit. These records were reviewed carefully by two investigators, in order not to miss any case. Records of all patients with bacterial meningitis were reviewed retrospectively to retrieve data on patients' demography, sign-symptoms, underlying medical conditions, investigations, names of microorganisms and their drug susceptibility, name and duration of therapy offered, appropriateness of therapy, and outcome.

2.5. Statistical Analysis. Quantitative variables were expressed as mean $\pm \mathrm{SD}$. Univariate logistic regression was performed to determine the probable predictors of inhospital mortality. All potential risk factors at $\leq 0.1$ level in the univariate analysis were entered in the multiple logistic regression to identify the independent predictors of mortality at $P<0.05$. The data were analyzed with SPSS software (v 17; IBM Corp., Armonk, NY, USA).

2.6. Ethical Approval. Ethical approval (\#13254/13) and a waiver of informed consent were obtained from the medical research ethical committee at Hamad Medical Corporation, Qatar.

\section{Results}

3.1. Demographic and Clinical Data. During the study period, we identified 117 episodes of ABM in 110 patients. There were $43,22,21,12$, and 18 episodes in 2009, 2010, 2011, 2012, and 2013, respectively. The study sample comprised 81 (69.2\%) male and $29(30.8 \%)$ female patients. Their mean age was $26.4 \pm 22.3$ years (range: $2-74$ ), and 28 (23.9\%) patients were Qatari. The peak frequency of ABM episodes was noted among adults (15-64 years old) (92.3\%) (see Table 1). From a clinical point of view, fever was the most frequent symptom $(110,94 \%)$, followed by mental alteration $(55,47 \%)$, headache $(43,36.8 \%)$, and vomiting $(35,29.9 \%)$. Moreover, meningismus was detected in 31 (26.5\%) patients (see Table 1).

3.2. Underlying Conditions. The most frequent underlying conditions were neurosurgery $(54,46.2 \%)$, hypertension $(26$, $22.2 \%)$, and diabetes mellitus (9,7.7\%) (see Table 1 ). 
TABLE 1: Demographic and clinical data of the 117 patients involved in this study.

\begin{tabular}{|c|c|}
\hline Variable & Number (\%), mean \pm SD (range) \\
\hline \multicolumn{2}{|l|}{ Gender } \\
\hline M & $81(69.2)$ \\
\hline $\mathrm{F}$ & $29(30.8)$ \\
\hline Age $($ mean $\pm \mathrm{SD})$, years & $26.4 \pm 22.3(2-74)$ \\
\hline \multicolumn{2}{|l|}{ Age group (years) } \\
\hline$<1$ & $26(22.2)$ \\
\hline $1-5$ & $11(9.4)$ \\
\hline $6-14$ & $6(5.1)$ \\
\hline $15-24$ & $12(10.3)$ \\
\hline $25-34$ & $13(11.1)$ \\
\hline $35-44$ & $17(14.5)$ \\
\hline $45-54$ & $23(19.7)$ \\
\hline $55-64$ & $6(5.1)$ \\
\hline$\geq 65$ & $3(2.6)$ \\
\hline \multicolumn{2}{|l|}{ Nationality } \\
\hline Qatari & $28(23.9)$ \\
\hline Non-Qatari & $82(76.1)$ \\
\hline \multicolumn{2}{|l|}{ Underlying conditions } \\
\hline Diabetes mellitus & $9(7.7)$ \\
\hline Hypertension & $26(22.2)$ \\
\hline Head injury & $6(5.1)$ \\
\hline Neurosurgery & $54(46.2)$ \\
\hline Alcoholic & $4(3.4)$ \\
\hline Prematurity & $7(6.0)$ \\
\hline Liver cirrhosis & $1(0.9)$ \\
\hline Otitis media & $5(4.3)$ \\
\hline Malignancy & $8(6.8)$ \\
\hline Immunosuppression & $2(1.7)$ \\
\hline \multicolumn{2}{|l|}{ Clinical presentation } \\
\hline Fever & $110(94)$ \\
\hline Mental alteration & $55(47)$ \\
\hline Headache & $43(36.8)$ \\
\hline Vomiting & $35(29.9)$ \\
\hline Meningism & $31(26.5)$ \\
\hline Seizures & $23(19.7)$ \\
\hline Bulging fontanel & $16(13.7)$ \\
\hline Hypotension $(\mathrm{BP}<90 / 60 \mathrm{mmHg})$ & $12(10.2)$ \\
\hline Focal signs & $11(9.4)$ \\
\hline Photophobia & $7(6.0)$ \\
\hline Behavioral changes & $3(2.6)$ \\
\hline Petechial rash & $3(2.6)$ \\
\hline \multicolumn{2}{|l|}{ Complications } \\
\hline Hydrocephalus & $19(16.2)$ \\
\hline Ischemic stroke & $5(4.3)$ \\
\hline Brain abscess & $4(3.4)$ \\
\hline Subdural empyema & $1(0.9)$ \\
\hline Adrenal insufficiency & $1(0.9)$ \\
\hline Vasculitis & $1(0.9)$ \\
\hline
\end{tabular}

3.3. Cerebrospinal Fluid (CSF) Findings. The CSF findings of the 117 ABM episodes are listed in Table 2.

3.4. Setting of Infection and Types and Distributions of the Microorganisms. Fifty-nine episodes (50.4\%) were
TABLE 2: Clinical data of the 117 patients involved in this study.

\begin{tabular}{lc}
\hline Variable & Number (\%), mean \pm SD (range) \\
\hline Acquisition of infection & $59(50.4)$ \\
Community-acquired & $58(49.6)$ \\
Nosocomial & \\
CSF & $3880.4 \pm 8654.6(20-66000)$ \\
Cells/ $\mu \mathrm{L}$ & $74.1 \pm 26.1(1-99)$ \\
Neutrophils\% & $22.9 \pm 24.8(1-98)$ \\
Lymphocytes\% & $222.8 \pm 205.9(38-936)$ \\
Protein (g/dL) & $2.1 \pm 1.6(0.1-6)$ \\
Glucose (mmol/L) & $93(79.5)$ \\
Positive Gram stain & $112(95.7)$ \\
Positive culture & $23(19.7)$ \\
Positive latex agglutination & \\
Type of microorganism & $62(53)$ \\
Gram-positive & $55(47)$ \\
Gram-negative & $37(31.6)$ \\
Positive blood culture & \\
Antimicrobial therapy & $97(82.9)$ \\
Appropriate & $20(17.1)$ \\
Inappropriate & $14(12.0)$ \\
Outcome & $103(88.0)$ \\
Died &
\end{tabular}

community-acquired and the other 58 (49.6\%) were nosocomially acquired ABM (see Table 3 ). The causative pathogens of the 117 enrolled ABM episodes are listed in Tables 3 and 4. Gram-positive pathogens accounted for 62 (53\%) episodes and Gram-negative pathogens accounted for the other 55 (47\%). In general, the most common causative agent of $\mathrm{ABM}$ in our cohort was coagulase-negative staphylococci; however, among the 59 community-acquired meningitis cases, the most common etiological agent was Streptococcus pneumoniae, whereas coagulase-negative staphylococci species were the leading cause of nosocomially acquired ABM. Among the implicated Gram-positive pathogens, coagulase-negative staphylococci were the most common (20,17\%), followed by Streptococcus pneumoniae (19, 16.2\%). Among Gram-negative pathogens, Klebsiella pneumoniae was the most common $(12,10.2 \%)$ followed by Neisseria meningitidis (11, 9.4\%).

3.5. Trends of Antimicrobial Susceptibility. Details of antimicrobial susceptibility are shown in Tables 5 and 6. Among the Gram-positive cases, 3 (18.6\%) episodes of Streptococcus pneumoniae were resistant to ceftriaxone, while out of all coagulase-negative staphylococci isolates, 19 (95\%) were methicillin-resistant. Among the Gram-negative cases, 100\% of Chryseobacterium species were resistant to meropenem and colistin, while $63.3 \%$ of Acinetobacter species showed resistance to meropenem but none for colistin. All Pseudomonas spp. were sensitive to piperacillin-tazobactam and meropenem. Among Klebsiella isolates, 2 (16.6\%) were extended spectrum beta-lactamase (ESBL) producers, but all 
TABLE 3: Distribution of different isolates in relation to setting of acquisition of meningitis.

\begin{tabular}{|c|c|c|c|}
\hline \multirow[b]{2}{*}{ Microorganism } & \multicolumn{2}{|c|}{ Setting of acquisition } & \multirow[b]{2}{*}{ Total } \\
\hline & $\begin{array}{c}\text { Nosocomial } \\
N(\%)\end{array}$ & $\begin{array}{c}\text { Community-acquired } \\
N(\%)\end{array}$ & \\
\hline \multicolumn{4}{|l|}{ Gram-positive } \\
\hline Abiotrophia species & $1(100)$ & 0 & 1 \\
\hline Enterococcus faecalis & $5(71.4)$ & $2(28.6)$ & 7 \\
\hline Enterococcus gallinarum & $1(100)$ & 0 & 1 \\
\hline Gemella haemolysans & $1(100)$ & 0 & 1 \\
\hline Leuconostoc species & $1(100)$ & 0 & 1 \\
\hline Listeria monocytogenes & 0 & $3(100)$ & 3 \\
\hline Staphylococcus aureus & 0 & $1(100)$ & 1 \\
\hline Staphylococcus capitis & $2(100)$ & 0 & 2 \\
\hline Staphylococcus epidermidis & $14(87.5)$ & $2(12.5)$ & 16 \\
\hline Staphylococcus haemolyticus & $2(100)$ & 0 & 2 \\
\hline Streptococcus agalactiae & 0 & $3(100)$ & 3 \\
\hline Streptococcus bovis II & 0 & $1(100)$ & 1 \\
\hline Streptococcus intermedius & 0 & $1(100)$ & 1 \\
\hline Streptococcus milleri & 0 & $1(100)$ & 1 \\
\hline Streptococcus mitis & 0 & $1(100)$ & 1 \\
\hline Streptococcus pneumoniae & 0 & $19(100)$ & 19 \\
\hline Streptococcus salivarius & 0 & $1(100)$ & 1 \\
\hline \multicolumn{4}{|l|}{ Gram-negative } \\
\hline Acinetobacter baumannii & $8(100)$ & 0 & 8 \\
\hline Acinetobacter lwoffii & $2(66.7)$ & $1(33,3)$ & 3 \\
\hline Brucella spp. & 0 & $1(100)$ & 1 \\
\hline Chryseobacterium (Flavobacterium) meningosepticum & 0 & $1(100)$ & 1 \\
\hline Chryseobacterium indologenes & 0 & $1(100)$ & 1 \\
\hline Enterobacter aerogenes & $1(100)$ & 0 & 1 \\
\hline Enterobacter cloacae & $3(100)$ & 0 & 3 \\
\hline Escherichia coli & $3(75)$ & $1(25)$ & 4 \\
\hline Haemophilus influenzae & $1(50)$ & $1(50)$ & 2 \\
\hline Klebsiella pneumoniae ssp. pneumoniae & $7(58.3)$ & $5(41.7)$ & 12 \\
\hline Neisseria meningitides & 0 & $11(100)$ & 11 \\
\hline Pseudomonas aeruginosa & $5(100)$ & 0 & 5 \\
\hline Pseudomonas putida & $1(100)$ & 0 & 1 \\
\hline Salmonella group B & 0 & $1(100)$ & 1 \\
\hline Serratia marcescens & 0 & $1(100)$ & 1 \\
\hline Total & 58 & 59 & 117 \\
\hline
\end{tabular}

of them were sensitive to meropenem and colistin. Of the 11 episodes with Neisseria meningitides infection, rifampicin resistance was found in $2(25 \%)$. Multidrug resistance was observed in 38 (32.4\%) of all episodes.

3.6. Treatment and Outcome. Antimicrobial treatment was initiated for all patients. Ceftriaxone plus vancomycin combination was the most widely used antimicrobial treatment followed by meropenem. Empiric therapy was inappropriate in $20(17.1 \%)$ episodes. The crude in-hospital mortality in our study was $14(12 \%)$.

3.7. Univariate and Multivariate Logistic Regression Analysis of Factors Associated with Death. By the univariate analysis, the following variables were found to be probable predictors of in-hospital mortality: presence of underlying diseases, nosocomial infection, multidrug-resistant episodes, hypotension, mental alteration, and inappropriate treatments 
TABLE 4: Distribution of microorganisms in relation to the age group.

\begin{tabular}{|c|c|c|c|c|c|c|c|c|c|c|}
\hline \multirow{2}{*}{ Microorganism } & \multicolumn{9}{|c|}{ Age group } & \multirow{2}{*}{ Total } \\
\hline & $<1$ & $1-5$ & $6-14$ & $15-24$ & $25-34$ & $35-44$ & $45-54$ & $55-64$ & $\geq 65$ & \\
\hline Abiotrophia species & 0 & 0 & 0 & 0 & 0 & 1 & 0 & 0 & 0 & $1(0.8)$ \\
\hline Acinetobacter baumannii & 0 & 0 & 0 & 0 & 0 & 2 & 3 & 2 & 1 & $8(6.8)$ \\
\hline Acinetobacter lwoffii & 0 & 1 & 1 & 0 & 1 & 0 & 0 & 0 & 0 & $3(2.6)$ \\
\hline Brucella spp. & 0 & 0 & 0 & 0 & 0 & 0 & 1 & 0 & 0 & $1(0.8)$ \\
\hline $\begin{array}{l}\text { Chryseobacterium (Flavobacterium) } \\
\text { meningosepticum }\end{array}$ & 1 & 0 & 0 & 0 & 0 & 0 & 0 & 0 & 0 & $1(0.8)$ \\
\hline Chryseobacterium indologenes & 1 & 0 & 0 & 0 & 0 & 0 & 0 & 0 & 0 & $1(0.8)$ \\
\hline Enterobacter aerogenes & 1 & 0 & 0 & 0 & 0 & 0 & 0 & 0 & 0 & $1(0.8)$ \\
\hline Enterobacter cloacae & 0 & 0 & 0 & 0 & 0 & 1 & 2 & 0 & 0 & $3(2.6)$ \\
\hline Enterococcus faecalis & 2 & 1 & 0 & 0 & 0 & 1 & 2 & 1 & 0 & $7(5.9)$ \\
\hline Enterococcus gallinarum & 0 & 0 & 0 & 0 & 0 & 0 & 1 & 0 & 0 & $1(0.8)$ \\
\hline Escherichia coli & 2 & 1 & 0 & 0 & 0 & 1 & 0 & 0 & 0 & $4(3.4)$ \\
\hline Gemella haemolysans & 0 & 0 & 0 & 1 & 0 & 0 & 0 & 0 & 0 & $1(0.8)$ \\
\hline Haemophilus influenzae & 0 & 1 & 0 & 1 & 0 & 0 & 0 & 0 & 0 & $2(1.6)$ \\
\hline Klebsiella pneumoniae ssp. pneumoniae & 2 & 0 & 0 & 2 & 1 & 1 & 4 & 2 & 0 & $12(10.3)$ \\
\hline Leuconostoc species & 0 & 0 & 0 & 0 & 0 & 0 & 1 & 0 & 0 & $1(0.8)$ \\
\hline Listeria monocytogenes & 3 & 0 & 0 & 0 & 0 & 0 & 0 & 0 & 0 & $3(2.6)$ \\
\hline Neisseria meningitidis & 0 & 1 & 0 & 3 & 3 & 1 & 2 & 0 & 1 & $11(9.4)$ \\
\hline Pseudomonas aeruginosa (PSA) & 0 & 0 & 1 & 0 & 2 & 1 & 1 & 0 & 0 & $5(4.2)$ \\
\hline Pseudomonas putida & 0 & 0 & 0 & 0 & 1 & 0 & 0 & 0 & 0 & $1(0.8)$ \\
\hline Salmonella group B & 1 & 0 & 0 & 0 & 0 & 0 & 0 & 0 & 0 & $1(0.8)$ \\
\hline Serratia marcescens & 1 & 0 & 0 & 0 & 0 & 0 & 0 & 0 & 0 & $1(0.8)$ \\
\hline Staphylococcus aureus & 0 & 0 & 1 & 0 & 0 & 0 & 0 & 0 & 0 & $1(0.8)$ \\
\hline Staphylococcus capitis & 0 & 0 & 0 & 0 & 0 & 1 & 1 & 0 & 0 & $2(1.6)$ \\
\hline Staphylococcus epidermidis & 6 & 2 & 0 & 2 & 1 & 3 & 1 & 1 & 0 & $16(13.6)$ \\
\hline Staphylococcus haemolyticus & 0 & 0 & 0 & 0 & 1 & 1 & 0 & 0 & 0 & $2(1.6)$ \\
\hline Streptococcus agalactiae & 2 & 0 & 0 & 0 & 0 & 0 & 0 & 0 & 1 & $3(2.6)$ \\
\hline Streptococcus bovis II & 1 & 0 & 0 & 0 & 0 & 0 & 0 & 0 & 0 & $1(0.8)$ \\
\hline Streptococcus intermedius & 0 & 0 & 0 & 0 & 0 & 1 & 0 & 0 & 0 & $1(0.8)$ \\
\hline Streptococcus milleri & 0 & 0 & 0 & 1 & 0 & 0 & 0 & 0 & 0 & $1(0.8)$ \\
\hline Streptococcus mitis & 0 & 1 & 0 & 0 & 0 & 0 & 0 & 0 & 0 & $1(0.8)$ \\
\hline Streptococcus pneumoniae & 3 & 2 & 3 & 2 & 3 & 2 & 4 & 0 & 0 & $19(16.2)$ \\
\hline Streptococcus salivarius & 0 & 1 & 0 & 0 & 0 & 0 & 0 & 0 & 0 & $1(0.8)$ \\
\hline Total & $\begin{array}{c}26 \\
(22.2) \\
\end{array}$ & $11(9.4)$ & $6(5.1)$ & $12(10.3)$ & $13(11.1)$ & $17(14.5)$ & $23(19.7)$ & $6(5.1)$ & $3(2.6)$ & $\begin{array}{c}117 \\
(100) \\
\end{array}$ \\
\hline
\end{tabular}

(see Table 7). Only the presence of underlying diseases, hypotension, and inappropriate treatment were found to be independent predictors of mortality by multivariate logistic regression analysis (see Table 8 ).

\section{Discussion}

Acute bacterial meningitis is a serious disease which necessitates early diagnosis and aggressive therapy to improve prognosis. Regional information regarding demographic data of patients, associated underlying conditions, etiology, and antimicrobial susceptibility is essential for correct and timely management of this disorder. Our study was the first to attempt to determine the clinical picture and the spectrum of pathogens of bacterial meningitis in patients of all ages in Qatar.

This retrospective series revealed some observations that deserve attention: firstly, in contrast with the previous study [6], the trend was seen to decrease from 2009 to 2013. Among the total 117 episodes, $43(36.7 \%)$ were reported in the year 2009, which decreased to 18 (15.4\%) in 2013. Furthermore, the disease in our series predominantly affected adults rather than infants and young children. This picture is similar to what was found in west countries and it may be attributed 
TABLE 5: Antimicrobial resistance rate of Gram-positive CSF isolates.

\begin{tabular}{|c|c|c|c|c|c|c|c|c|c|c|c|c|}
\hline Microorganisms & TNP & pen & $\mathrm{amp}$ & oxc & eryt & clind & amclv & cotr & $\mathrm{cfr}$ & van & line & teic \\
\hline Abiotrophia spp. & 1 & 0 & 0 & NT & 0 & 0 & 0 & 0 & NT & 0 & NT & NT \\
\hline Enterococcus faecalis & 7 & NT & 0 & NT & NT & NT & NT & NT & NT & 0 & 0 & 0 \\
\hline Enterococcus gallinarum & 1 & NT & 0 & NT & NT & NT & NT & NT & NT & $1(100)$ & 0 & 0 \\
\hline Gemella haemolysans & 1 & 0 & 0 & NT & NT & NT & NT & NT & 0 & 0 & NT & NT \\
\hline Leuconostoc species & 1 & 0 & 0 & NT & NT & 0 & 0 & NT & $1(100)$ & $1(100)$ & 0 & 0 \\
\hline Listeria monocytogenes & 3 & 0 & 0 & NT & NT & NT & NT & 0 & NT & NT & NT & NT \\
\hline Staphylococcus aureus & 1 & $1(100)$ & $1(100)$ & NT & 0 & 0 & 0 & 0 & NT & 0 & 0 & 0 \\
\hline Coagulase-negative staphylococci & 20 & $20(100)$ & $20(100)$ & $19(95)$ & $19(95)$ & $10(50)$ & $20(100)$ & $9(45)$ & NT & 0 & 0 & 0 \\
\hline Streptococcus spp. & 8 & $1(0.2)$ & $1(0.2)$ & 0 & $1(0.2)$ & $1(0.2)$ & 0 & $1(0.2)$ & $1(0.2)$ & 0 & 0 & 0 \\
\hline Streptococcus pneumoniae & 19 & $6(31.5)$ & $6(31.5)$ & $6(31.5)$ & $6(31.5)$ & $6(31.5)$ & $6(31.5)$ & $3(15.7)$ & $3(15.7)$ & 0 & 0 & 0 \\
\hline
\end{tabular}

TNP: total number of patients; pen: penicillin; amp: ampicillin; oxc: oxacillin; eryt: erythromycin; clind: clindamycin; amclv: amoxicillin/clavulanic acid; cotr: cotrimoxazole; cfr: ceftriaxone; van: vancomycin; line: linezolid; teic: teicoplanin; NT: not tested.

TABLE 6: Antimicrobial resistance rate of Gram-negative cerebrospinal fluid isolates.

\begin{tabular}{|c|c|c|c|c|c|c|c|c|c|c|c|c|c|c|}
\hline Microorganisms & TNP & $\mathrm{cfr}$ & gen & fep & taz & cip & amclv & $\mathrm{ctz}$ & amk & mem & pen & rif & col & tig \\
\hline Acinetobacter spp. & 11 & $8(72.7)$ & $8(72.7)$ & $8(72.7)$ & $8(72.7)$ & $7(63.6)$ & NT & $\begin{array}{c}7 \\
(63.6)\end{array}$ & $\begin{array}{c}7 \\
(63.6)\end{array}$ & $\begin{array}{c}7 \\
(63.6)\end{array}$ & NT & NT & 0 & 0 \\
\hline Brucella species & 1 & NT & NT & NT & NT & NT & NT & NT & NT & NT & NT & NT & NT & NT \\
\hline $\begin{array}{l}\text { Chryseobacterium } \\
\text { spp. }\end{array}$ & 2 & $1(50)$ & $2(100)$ & 0 & $1(50)$ & 0 & NT & $2(100)$ & $2(100)$ & $2(100)$ & NT & NT & $2(100)$ & $\begin{array}{c}2 \\
(100)\end{array}$ \\
\hline Enterobacter species & 4 & $3(75)$ & 0 & $1(25)$ & $3(75)$ & 0 & $4(100)$ & $3(75)$ & 0 & 0 & NT & NT & NT & NT \\
\hline Escherichia coli & 4 & $1(25)$ & $1(25)$ & $1(25)$ & 0 & $1(25)$ & $1(25)$ & $1(25)$ & 0 & 0 & NT & NT & NT & NT \\
\hline $\begin{array}{l}\text { Haemophilus } \\
\text { influenzae }\end{array}$ & 2 & 0 & NT & 0 & 0 & NT & 0 & NT & NT & NT & NT & NT & NT & NT \\
\hline $\begin{array}{l}\text { Klebsiella pneumoniae } \\
\text { ssp. pneumoniae }\end{array}$ & 12 & $3(25)$ & 0 & $3(25)$ & $2(16.6)$ & $2(16.6)$ & $\begin{array}{c}7 \\
(58.3)\end{array}$ & $\begin{array}{c}4 \\
(33.3)\end{array}$ & 0 & 0 & NT & NT & 0 & 0 \\
\hline Neisseria meningitidis & 8 & 0 & NT & 0 & 0 & $2(25)$ & 0 & NT & NT & 0 & 0 & $2(25)$ & NT & NT \\
\hline $\begin{array}{l}\text { Pseudomonas } \\
\text { aeruginosa }\end{array}$ & 5 & NT & 0 & 0 & 0 & 0 & NT & 0 & 0 & 0 & NT & NT & 0 & 0 \\
\hline Pseudomonas putida & 1 & NT & 0 & 0 & 0 & 0 & NT & 0 & 0 & 0 & NT & NT & 0 & 0 \\
\hline Salmonella group B & 1 & 0 & $1(100)$ & 0 & 0 & 0 & 0 & 0 & 0 & 0 & NT & NT & NT & NT \\
\hline Serratia marcescens & 1 & 0 & 0 & 0 & 0 & 0 & $1(100)$ & 0 & 0 & 0 & NT & NT & NT & NT \\
\hline
\end{tabular}

TNP: total number of patients; cfr: ceftriaxone; gen: gentamicin; fep: cefepime; taz: piperacillin/tazobactam; cip: ciprofloxacin; amclv: amoxicillin/clavulanic acid; ctz: ceftazidime; amk: amikacin; mem: meropenem; pen: penicillin; rif: rifampicin; col: colistin; tig: tigecycline; NT: not tested.

TABLE 7: Results of univariate analysis of in-hospital mortality predictors.

\begin{tabular}{lcc}
\hline Variable & $\begin{array}{c}\text { Unadjusted odds ratio } \\
(95 \% \text { CI })\end{array}$ & $P$ value \\
\hline $\begin{array}{l}\text { Presence of underlying } \\
\text { diseases }\end{array}$ & $2.4(1.4-3.9)$ & 0.001 \\
$\begin{array}{l}\text { Nosocomial infection } \\
\text { Multidrug-resistant }\end{array}$ & $3.2(1.5-6.5)$ & 0.1 \\
$\begin{array}{l}\text { episodes } \\
\text { Mental alteration }\end{array}$ & $4.7(3.8-5.7)$ & 0.08 \\
$\begin{array}{l}\text { Hypotension } \\
\text { Inappropriate treatments }\end{array}$ & $4.9(1.0-24.0)$ & 0.06 \\
\hline
\end{tabular}

to vaccine-related decline in $H$. influenzae and pneumococcal diseases $[3,9,14,15]$. These data show that adults are the main
TABLE 8: Results of multivariate analysis of in-hospital mortality independent factors.

\begin{tabular}{lcc}
\hline Variable & $\begin{array}{l}\text { Adjusted odds } \\
\text { ratio (95\% CI) }\end{array}$ & $P$ value \\
\hline $\begin{array}{l}\text { Presence of underlying } \\
\text { diseases }\end{array}$ & $1.8(1.03-3.2)$ & 0.02 \\
$\begin{array}{l}\text { Hypotension } \\
\text { Inappropriate treatments }\end{array}$ & $3.2(1.4-7.3)$ & 0.04 \\
\hline
\end{tabular}

target population which requires interventions to prevent and control diseases in Qatar.

Secondly, sex distribution of the disease showed male predominance in agreement with the previous report [6] and other reports from different countries [9, 10, 16-19]. The 
reason for this is obscure, and further studies are needed to identify the cause.

Thirdly, compared with the previous studies $[4,6]$, changes of common causative pathogens of ABM had been noted in our series. Coagulase-negative staphylococci species were the most common causative agents followed by Streptococcus pneumoniae. This can be explained by the expansion of neurosurgical services in our hospital with a consequent increase in the number of patients with postneurosurgical state. Similarly, reports from Taiwan [15-18] showed that there has been an increasing incidence of staphylococcal infection in ABM patients. However, in agreement with many reports worldwide [2, 7, 20-23], Streptococcus pneumoniae remain the common causative agent for community-acquired infection in our study.

Fourthly, drug resistance pattern showed that 95\% of the implicated coagulase-negative staphylococci species were oxacillin-resistant and $63.3 \%$ of the implicated Acinetobacter species were meropenem-resistant. Both infections were predominantly nosocomial, which raised doubt regarding the infection control program in our hospital. Moreover, these findings result in therapeutic challenge in the choice of empiric antibiotics in the initial management of ABM. These findings are consistent with reports coming from Taiwan recently $[9,18,19]$. Fortunately, so far, we have not encountered vancomycin-resistant coagulase-negative staphylococci strains or colistin-resistant Acinetobacter strains.

Finally, in an attempt to identify independent predictors of mortality in patients with ABM, many studies had been conducted. The concluded prognostic factors among these studies were diverse $[9,10,18,19,22,23]$. Our study revealed many probable prognostic factors; however, only the presence of underlying diseases, hypotension, and inappropriate treatment were found to be independent predictors of mortality by multivariate logistic regression analysis.

This hospital-based study has the following limitations. First, the study was retrospective rather than prospective, and this design did not allow us to obtain additional details such as severity of the disease and long-term follow-up to evaluate the long-term sequelae of meningitis in our patients. Second, it was performed at a single hospital; the results may not be applicable to other hospitals. Third, we included patients who had a positive CSF culture or positive CSF bacterial antigen test.

Despite these limitations, we believe that our study remains the largest to date to provide comprehensive information on the epidemiology of ABM in Qatar.

In conclusion, our study revealed that there is a change in the predominantly affected age group and common causative agents of ABM. Coagulase-negative staphylococci species are the common causative agent in Qatar with majority of infections occurring nosocomially. More than $90 \%$ of all implicated coagulase-negative staphylococci strains were oxacillin-resistant. Thus, improving our infection control programs in addition to enhancing antimicrobial stewardship is essential to overcome this problem.

\section{Conflicts of Interest}

The authors declare that they have no conflicts of interest.

\section{References}

[1] R. Miller and K. Houlberg, "Meningitis service-age personnel," Journal of the Royal Naval Medical Service, vol. 101, pp. 63-68, 2015.

[2] E. S. Gurley, M. J. Hossain, S. P. Montgomery et al., "Etiologies of bacterial meningitis in Bangladesh: results from a hospitalbased study," The American Journal of Tropical Medicine and Hygiene, vol. 81, no. 3, pp. 475-483, 2009.

[3] M. C. Brouwer, A. R. Tunkel, and D. van de Beek, "Epidemiology, diagnosis, and antimicrobial treatment of acute bacterial meningitis," Clinical Microbiology Reviews, vol. 23, no. 3, pp. 467-492, 2010.

[4] M. F. Elsaid, H. Alsoub, M. S. Bessisso et al., "Clinical presentation of acute bacterial meningitis in Qatar," Neurosciences, vol. 7, no. 4, pp. 266-271, 2002.

[5] M. F. Elsaid, A. A. Flamerzi, M. S. Bessisso, and S. S. Elshafie, "Acute bacterial meningitis in Qatar," Saudi Medical Journal, vol. 27, no. 2, pp. 198-204, 2006.

[6] S. F. Shah, S. Nadeem, A. Farooq, and A. Bener, "Emerging trend in the epidemiology of meningitis in Qatar," Journal of the College of Physicians and Surgeons-Pakistan, vol. 18, pp. 259-260, 2008.

[7] M. L. Durand, S. B. Calderwood, and D. J. Weber, "Acute bacterial meningitis in adults: a review of 493 episodes," The New England Journal of Medicine, vol. 328, no. 1, pp. 21-28, 1993.

[8] B. Sigurdardóttir, Ó. M. Björnsson, K. E. Jónsdóttir, H. Erlendsdóttir, and S. Gudmundsson, "Acute bacterial meningitis in adults: a 20-year overview," Archives of Internal Medicine, vol. 157, no. 4, pp. 425-430, 1997.

[9] W.-N. Chang, C.-H. Lu, C.-R. Huang et al., "Changing epidemiology of adult bacterial meningitis in Southern Taiwan: a hospital-based study," Infection, vol. 36, no. 1, pp. 15-22, 2008.

[10] H. Georges, A. Chiche, S. Alfandari, P. Devos, N. Boussekey, and O. Leroy, "Adult community-acquired bacterial meningitis requiring ICU admission: epidemiological data, prognosis factors and adherence to IDSA guidelines," European Journal of Clinical Microbiology and Infectious Diseases, vol. 28, no. 11, pp. 1317-1325, 2009.

[11] F. Y. Khan, M. Abukhattab, and K. Baager, "Nosocomial postneurosurgical acinetobacter baumannii meningitis: a retrospective study of six cases admitted to hamad general hospital, Qatar," Journal of Hospital Infection, vol. 80, no. 2, pp. 176-179, 2012.

[12] Management of multidrug-resistant organisms in healthcare settings, 2006, Centers for Disease Control and Prevention, 2016, https://www.cdc.gov/ncidod/dhqp/pdf/ar/mdroGuideline2006 .pdf.

[13] P. A. Wayne, "Performance standards for antimicrobial susceptibility testing: twenty-first informational supplement," Clinical and Laboratory Standards Institute (CLSI), pp. M100-S24, 2014.

[14] N. G. Martin, M. Sadarangani, A. J. Pollard, and M. J. Goldacre, "Hospital admission rates for meningitis and septicaemia caused by Haemophilus influenzae, Neisseria meningitidis, and Streptococcus pneumoniae in children in England over five decades: a population-based observational study," The Lancet Infectious Diseases, vol. 14, no. 5, pp. 397-405, 2014. 
[15] M. A. Dery and R. Hasbun, "Changing epidemiology of bacterial meningitis," Current Infectious Disease Reports, vol. 9, no. 4, pp. 301-307, 2007.

[16] Y. Xie, Y. Tan, V. Chongsuvivatwong et al., "A population-based acute meningitis and encephalitis syndromes surveillance in Guangxi, China, May 2007- June 2012," PLoS ONE, vol. 10, no. 12, article e0144366, 2015.

[17] C. Huang, C. Lu, J. Wu et al., "Coagulase-negative staphylococcal meningitis in adults: clinical characteristics and therapeutic outcomes," Infection, vol. 33, no. 2, pp. 56-60, 2005.

[18] W. N. Chang, C. H. Lu, C. R. Huang et al., "Epidemiology of adult staphylococcal meningitis southern Taiwan: a of infection and coagulase-negative staphylococcal infection," Japanese Journal of Infectious Diseases, vol. 60, no. 5, pp. 262-266, 2007.

[19] M.-H. Tsai, C.-H. Lu, C.-R. Huang et al., "Bacterial meningitis in young adults in southern Taiwan: clinical characteristics and therapeutic outcomes," Infection, vol. 34, no. 1, pp. 2-8, 2006.

[20] M. M. Abdelkader, K. M. Aboshanab, M. A. El-Ashry, M. M. Aboulwafa, and S. D. Sekaran, "Prevalence of MDR pathogens of bacterial meningitis in Egypt and new synergistic antibiotic combinations," PLOS ONE, vol. 12, no. 2, article e0171349, 2017.

[21] H. Erleena Nur, I. Jamaiah, M. Rohela, and V. Nissapatorn, "Bacterial meningitis: a five year (2001-2005) retrospective study," in The Southeast Asian Journal of Tropical Medicine and Public Health, vol. 39, pp. 73-77, University Malaya Medical center (UMMC), Kuala Lumpur, Malaysia, 2008.

[22] O. Dzupova, H. Rozsypal, B. Prochazka, and J. Benes, "Acute bacterial meningitis in adults: predictors of outcome," Scandinavian Journal of Infectious Diseases, vol. 41, no. 5, pp. 348-354, 2009.

[23] D. van de Beek, J. de Gans, L. Spanjaard, M. Weisfelt, J. B. Reitsma, and M. Vermeulen, "Clinical features and prognostic factors in adults with bacterial meningitis," The New England Journal of Medicine, vol. 351, no. 18, pp. 1849-1859, 2004. 


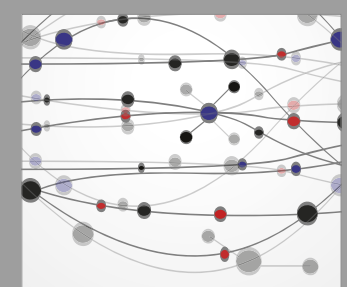

The Scientific World Journal
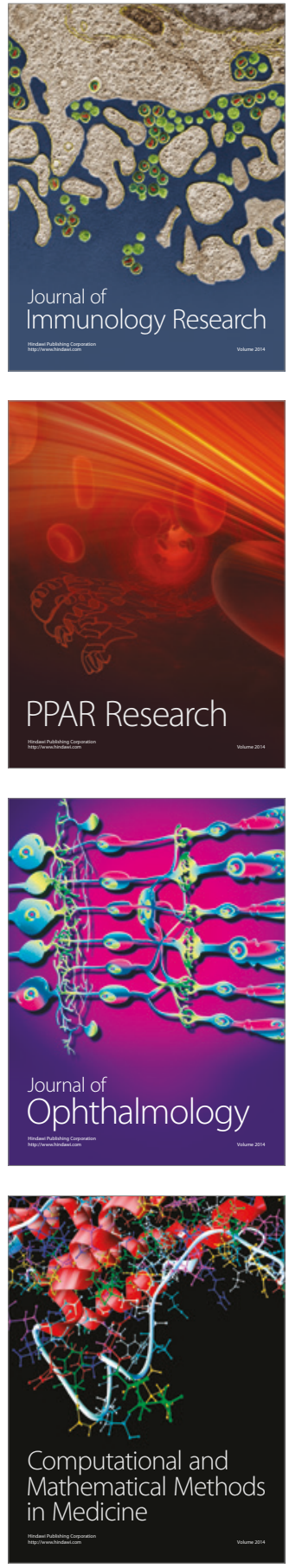

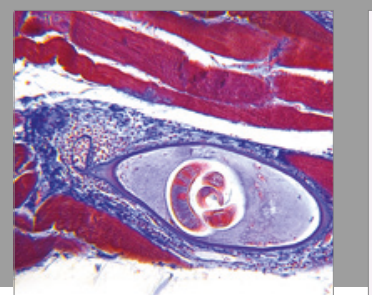

Gastroenterology Research and Practice
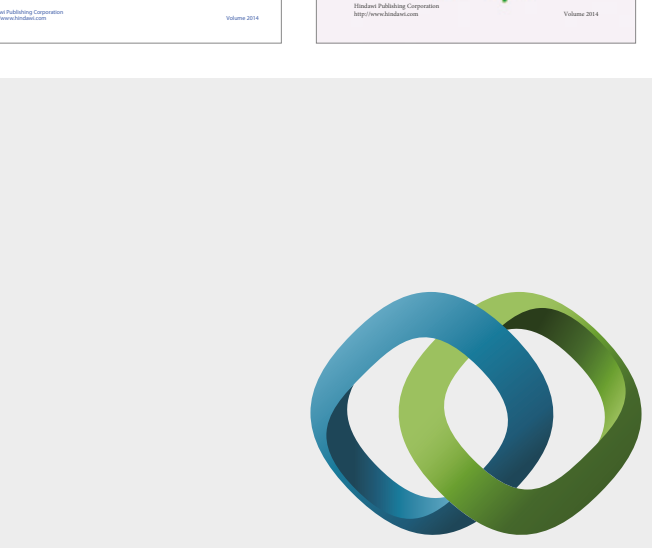

\section{Hindawi}

Submit your manuscripts at

https://www.hindawi.com
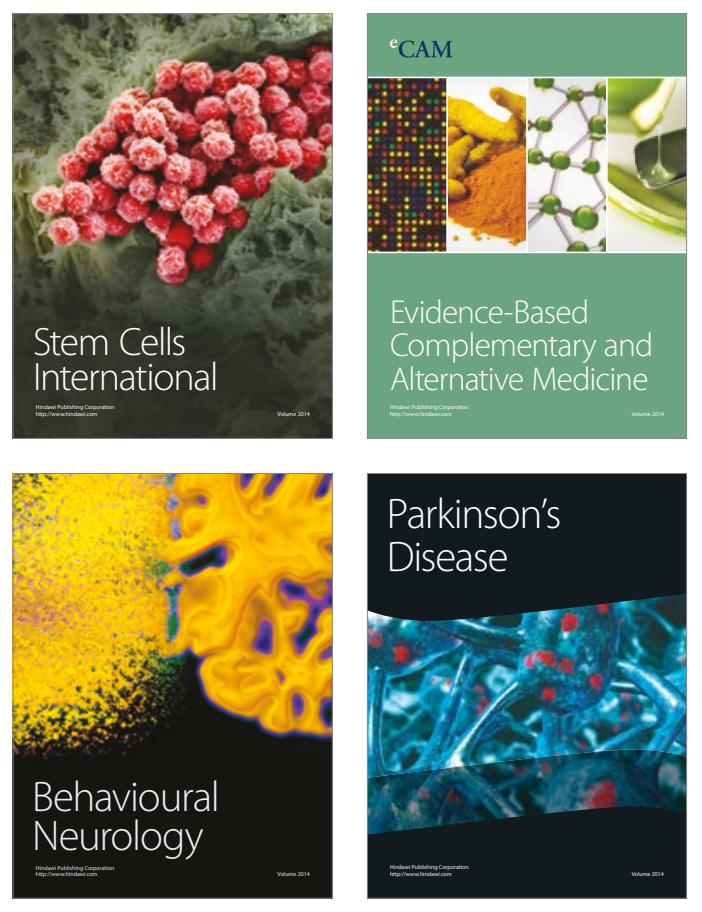
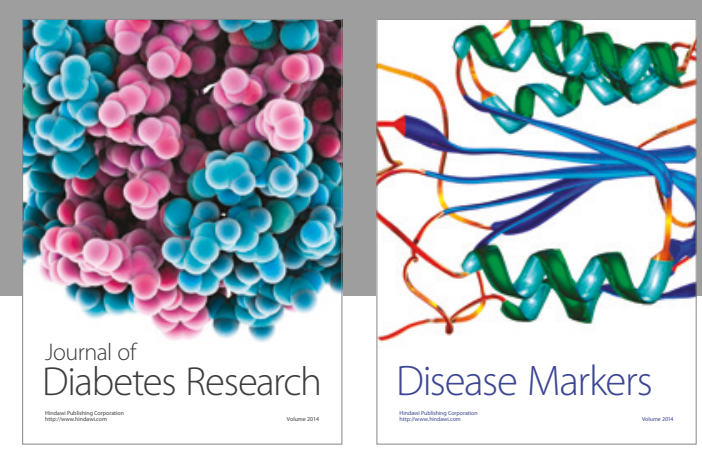

Disease Markers
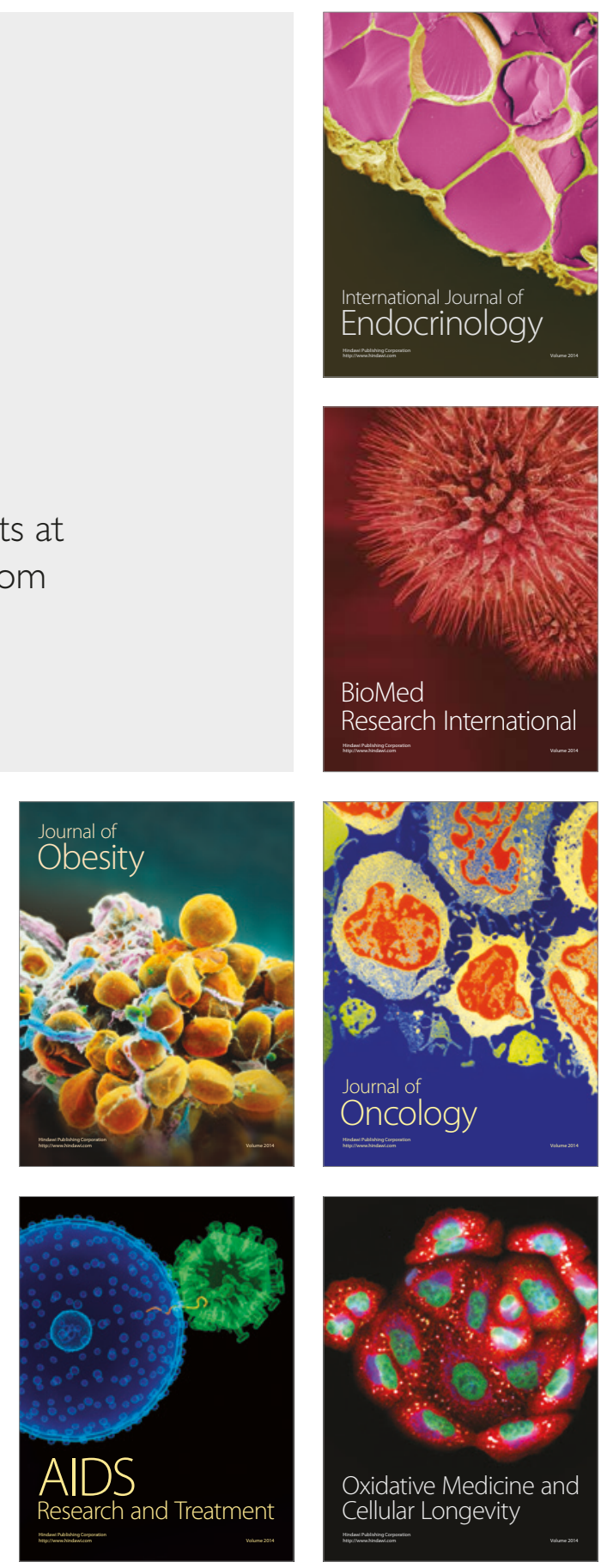